\title{
Understanding BL Lacertae objects
}

\section{Structural and kinematic mode changes in the BL Lac object PKS 0735+178}

\author{
S. Britzen ${ }^{1}$, A. Witzel ${ }^{1}$, B. P. Gong ${ }^{2}$, J. W. Zhang ${ }^{2}$, G. Krishna ${ }^{3}$, A. Goyal ${ }^{4}$, M. F. Aller ${ }^{5}$, H. D. Aller ${ }^{5}$, and \\ J. A. Zensus ${ }^{1}$
}

\begin{abstract}
1 Max-Planck-Institut für Radioastronomie, Auf dem Hügel 69, 53121 Bonn, Germany e-mail: sbritzen@mpifr-bonn.mpg.de

2 Department of Physics, Huazhong University of Science and Technology, Wuhan 430074, PR China

3 National Centre for Radio Astrophysics/TIFR, Post Bag 3, Pune University Campus, Pune, 411007, India

4 Aryabhatta Research Institute of observational sciencES (ARIES), Manora Peak, Naini Tal 263 129, India

5 Astronomy Department, University of Michigan, Ann Arbor, MI 48109-1042, USA
\end{abstract}

Received 17 November 2009 / Accepted 26 January 2010

\section{ABSTRACT}

Context. We present evidence that parsec-scale jets in BL Lac objects may be significantly distinct in kinematics from their counterparts in quasars. We argued this previously for the BL lac sources $1803+784$ and $0716+714$ and report here a similar pattern for another well-known BL Lac object, PKS 0735+178, whose nuclear jet is found to exhibit kinematics atypical of quasars.

Aims. By analyzing the pc-scale jet morphology and its changes in 0735+178 we seek to understand the emission processes in BL Lac objects and to decipher their differences from quasars. A detailed study of the jet components' motion reveals that the standard AGN paradigm of apparent superluminal motion does not always describe the kinematics in BL Lac objects. We study $0735+178$ here to augment and improve the understanding of the peculiar motions in the jets of BL Lac objects as a class.

Methods. We analyzed $15 \mathrm{GHz}$ VLBA (Very Long Baseline Array) observations ( $2 \mathrm{~cm} / \mathrm{MOJAVE}$ survey) performed at 23 epochs between 1995.27 and 2008.91. Multiple Gaussians were fitted to the derived VLBA data, to trace the kinematical and flux density evolution of the individual VLBI (Very Long Baseline Interferometry) components in the nuclear jet. We then compared the jet kinematics with the optical and radio light curves available for this BL Lac object and point out some striking correlations between the properties of the radio knots and the features in the light curves.

Results. We found a drastic structural mode change in the VLBI jet of 0735+178, between 2000.4 and 2001.8 when its twice sharply bent trajectory turned into a linear shape. We further found that this jet had undergone a similar transition sometime between December 1981 and June 1983. A mode change, occurring in the reverse direction (between mid-1992 and mid-1995) has already been reported in the literature. These structural mode changes are found to be reflected in changed kinematical behavior of the nuclear jet, manifested as an apparent superluminal motion and stationarity of the radio knots. In addition, we found the individual mode changes to correlate in time with the maxima in the optical light curve. The last two transitions occurred before a (modest) radio flare. The behavior of this pc-scale jet appears to favor a scenario involving non-ballistic motions of the radio knots, produced by the precession of a continuous jet within the ambient medium.

Conclusions. This is the third BL Lac object (after 1803+784 and 0716+714) analyzed by us, which reveals kinematic properties in the parsec-scale radio jet, which are atypical of quasars. For several years, the components in the jet are not found to be separating from the core but instead to be moving perpendicularly to the ridge line of the jet. In 0735+178 this unusual behavior has been particularly conspicuous. Curiously, however, fast apparent superluminal motion characteristic of quasars were present in this jet between the epochs $\sim 1995$ and $\sim 2000$. Thus we found for this BL Lac object a drastic change in the kinematics of the nuclear jet, i.e, transition from "typical superluminal" to an unusual "stationary" state. Interestingly, we found that this change is accompanied with a mode change in the nuclear jet's morphology. The long sequences of VLBA images reported here for this BL Lac object do not support the commonly assumed connection between radio flux-density outbursts and the ejection of new VLBI components. In contrast, the two mode changes seem correlated with the radio flares and are more likely an outcome of a changing viewing angle of the same knots. We propose therefore that all these observed features of the nuclear jet of $0735+178$ and their correlation with the optical and radio flares might hold significant promise to better understand the basic difference between BL Lac objects and quasars.

Key words. BL Lacertae objects: individual: PKS 0735+178 - techniques: interferometric - radio continuum: galaxies

\section{Introduction}

The strong radio source PKS $0735+178$ is an optically bright classical BL Lac object, highly variable at radio/optical wavelengths (e.g., Goyal et al. 2009 and references therein). Based on an absorption feature in the optical spectrum, a lower redshift limit of 0.424 was established (Abraham et al. 1991; Stickel et al. 1993; Scarpa et al. 2000). This is consistent with the non detection of the optical host in the HST snapshot imaging survey by Sbarufatti et al. (2005). A companion (possible absorber) has been detected at a projected separation of $22 \mathrm{kpc}$ (Pursimo et al. 1999). Extensive monitoring of this blazar in the radio and optical bands revealed variability on different timescales. Periodic features of 13.8-14.2 years were found by applying the Jurkevich method to about a century long optical monitoring data (Fan et al. 1997; Qian \& Tao 2004). 
Ciprini et al. (2007) reported possible detection of several shorter time scales. In their search for intra-night optical variability on 17 nights between 1998 and 2008, Goyal et al. (2009) did not detect on any occasion intra-night variability with an amplitude $\geq 3 \%$, though such amplitudes are typical for radio selected BL Lacs (Gopal-Krishna et al. 2003). Gupta et al. (2008) claimed detection of optical variability with an amplitude of $4.9 \%$ on one night, but since that light curve remains steady, any such variability must be of the flicker type and not on hour-like time scale.

In the radio band, an unusual property highlighted for this BL Lac is its remarkably flat radio spectrum, termed as "cosmic conspiracy" (Cotton et al. 1980).

\subsection{Radio morphology: kpc- to pc-scale}

PKS $0735+178$ has been observed with radio interferometers at various frequencies. The 1.64 GHz VLA (Very Large Array) observations by Murphy et al. (1993) revealed a rather compact structure, slightly elongated at a position angle of $\sim 160 \mathrm{deg}$. The $5 \mathrm{GHz}$ MERLIN (Multi-Element Radio Linked Interferometer Network) image taken in 1983 is dominated by a core and also shows a very weak component to the southeast (Bååth \& Zhang 1991). The core-to-component flux density ratio is 200:1 and the position angle of the component is $\simeq 160 \mathrm{deg}-$ in agreement with the VLA observations by Murphy et al. (1993). These observations established a large misalignment between the kpc-scale and pc-scale structures of this source by $\sim 90-100 \mathrm{deg}$.

PKS $0735+178$ has been a favorite target of VLBI monitoring programs, and striking changes in its nuclear jet are revealed from the more than two decades of its VLBI monitoring.

Bååth \& Zhang (1991) imaged the source at $5 \mathrm{GHz}$ with global VLBI arrays at four epochs between 1979 and 1984. The large structural changes thus found were interpreted by them in terms of relativistic $(\gamma \sim 8)$ motion of the radio knots along a curly path with a steadily decreasing viewing angle. Particularly instructive are their VLBI images of the inner 5 mas region, taken at the epochs 1981.9 and 1983.5, which (despite modest resolution) reveal a clear changeover from a kinky staircase profile in 1981.9 to a straight mode in 1983.5 (see below). This morphological transition, first noted in the present work, is discussed in Sect. 3.

Gabuzda et al. (1994) observed the source with the VLBA at the epochs 1987.41 (5 GHz), $1990.47(8.4 \mathrm{GHz})$, and 1992.44 $(5 \mathrm{GHz})$. In all these observations, the source revealed a straight jet extending to the northeast. Gómez et al. (2001) present VLBA observations at 8.4 and $43 \mathrm{GHz}$ between August 1996 and May 1998. Between March 1996 and May 2000 Agudo et al. (2006) observed the source using the VLBA at frequencies between 5 and $43 \mathrm{GHz}$. The observations by Gómez et al. and Agudo et al. show a twisted jet with two sharp apparent bends of $90 \circ$ within two milli-arcseconds of the core. Below we call this trajectory staircase mode. Between mid-1992 and mid 1995, the jet trajectory changed again drastically from straight to staircase (Gómez et al. 2001). Unfortunately, no VLBI observations documenting this second transition are available.

From early VLBI observations of this BL Lac object Bååth \& Zhang (1991) estimated bulk Lorentz factors of up to $\sim 8 h^{-1} c$ for the knots in the VLBI jet, moving from the core towards a stationary component located $\sim 4$ millisecond northeast of the core. A subsequent paper by Gabuzda et al. (1994) reports an apparent superluminal motion of $\geq 7.4 h^{-1} c$ of one knot. After mid-1995 the components show a tendency to cluster near several jet positions, remaining stationary in time (Sects. 3 and 4.2).

\subsection{The present work}

The extensive VLBA coverage of this blazar at $15 \mathrm{GHz}$ during 1995-2009 under the MOJAVE program allowed us to undertake a detailed probe of structural transitions in the nuclear jet of this BL Lac object (Sect. 3). Thus, in addition to the last documented mode change in the pc-scale trajectory (Gómez et al. 2001), we found clear evidence for another mode change between 2000.4 and 2001.8. We find that from the staircase mode observed since mid 1995 the jet trajectory has reverted to a straight morphology during the time interval covered by our VLBA data analysis, so that the timing of this morphological change can be pinned down to an uncertainty of merely $\sim 1.6$ years. In comparison, the uncertainty for the mode change inferred from the data of Gómez et al. was $\sim 3$ years. According to them, the morphological transitions are most likely caused by pressure gradients in the external medium through which the jet propagates. We discuss this hypothesis with regard to the mode transitions found by us. We further examine these mode transitions gleaned from the VLBI data, as mentioned above, with regard to the observed trends in the radio and optical light-curves of this intensively monitored blazar.

This paper is organized in the following way: we first present the results of our analysis of the archival VLBA data (MOJAVE) and point out the newly noticed correlations between the morphological and flux-density variations of this source. We then discuss our findings in the context of previously reported information about this blazar. In particular, we explore the connection between the peculiar mode changes and kinematics of its nuclear jet. Apparent transverse velocities $\left(\beta_{\text {app }}\right)$ in this paper were calculated with the one-year WMAP (Wilkinson Microwave Anisotropy Probe) data (Spergel et al. 2003); the values adopted are $h=0.71_{-0.03}^{+0.04} ; \Omega_{m} h^{2}=0.135_{-0.009}^{+0.008} ; \Omega_{\text {tot }}=1.02 \pm 0.2$. The one-year and three-year WMAP parameters are less than $0.1 c$ for $\mu=0.1$ mas $\mathrm{yr}^{-1}$ out to $z=4$; this is small with respect to the formal uncertainties in the apparent velocities.

\section{Data analysis}

We analyzed the VLBA observations for 23 epochs, taken at $15 \mathrm{GHz}$ under the $2 \mathrm{~cm} /$ MOJAVE survey between 1995 and 2009. The data were model-fitted with the difmap model-fitting routine. To each epoch's VLBA image we attempted to fit the minimum number of components needed to describe the structure adequately to facilitate a least ambiguous component identification from epoch to epoch. For each image the model-fitting process started with a point-like source model. In order to keep the comparison of the images for different epochs simple and credible, we assumed the components to be circular; highly elliptical components are not likely anyway.

\subsection{Estimation of the uncertainties}

Although the use of VLBA data generally ensures a high quality, considerable variation can occur between the epochs in both the amount of data and its quality. The uncertainties were determined individually for each epoch by comparing the modelfit parameters for the sets with different numbers of components $( \pm 1)$. For the uncertainties of the flux-density we assumed a minimum error of $5 \%$. The positional uncertainty was determined by averaging $1 / 5$ th of the beam size and the value determined by comparing several model-fit results. For the size we adopted an uncertainty of $10 \%$. The model fit parameters and their uncertainties are given in Table 1. We also list there the epoch of 
Table 1. Model-fit results.

\begin{tabular}{|c|c|c|c|c|c|}
\hline Epoch & Flux-density [Jy] & Core sep. [mas] & Pos. a. [deg] & Maj. a. [mas] & Id. \\
\hline \multirow[t]{4}{*}{1995 Apr. 7} & $0.956 \pm 0.047$ & $0.00 \pm 0.00$ & $0.0 \pm 0.0$ & $0.17 \pm 0.02$ & $\mathrm{k}$ \\
\hline & $0.537 \pm 0.038$ & $0.63 \pm 0.09$ & $80.0 \pm 5.4$ & $0.37 \pm 0.04$ & $\mathrm{c} 1$ \\
\hline & $0.098 \pm 0.005$ & $1.25 \pm 0.10$ & $38.3 \pm 2.6$ & $0.96 \pm 0.10$ & c3 \\
\hline & $0.051 \pm 0.003$ & $4.35 \pm 0.06$ & $62.7 \pm 2.5$ & $1.47 \pm 0.15$ & c5 \\
\hline \multirow[t]{6}{*}{1995 Dec. 15} & $0.557 \pm 0.028$ & $0.00 \pm 0.00$ & $0.0 \pm 0.0$ & $0.19 \pm 0.02$ & $\mathrm{k}$ \\
\hline & $0.275 \pm 0.014$ & $0.62 \pm 0.05$ & $94.3 \pm 2.5$ & $0.32 \pm 0.03$ & $\mathrm{c} 1$ \\
\hline & $0.196 \pm 0.010$ & $0.91 \pm 0.05$ & $70.1 \pm 2.7$ & $0.33 \pm 0.03$ & $\mathrm{c} 2$ \\
\hline & $0.099 \pm 0.005$ & $1.00 \pm 0.05$ & $37.7 \pm 2.9$ & $0.73 \pm 0.07$ & c3 \\
\hline & $0.040 \pm 0.002$ & $2.25 \pm 0.08$ & $35.1 \pm 3.0$ & $1.18 \pm 0.12$ & $\mathrm{c} 4$ \\
\hline & $0.018 \pm 0.001$ & $4.33 \pm 0.07$ & $62.6 \pm 2.7$ & $0.74 \pm 0.07$ & $\mathrm{c} 5$ \\
\hline \multirow[t]{5}{*}{1996 Jan. 19} & $0.565 \pm 0.028$ & $0.00 \pm 0.00$ & $0.0 \pm 0.0$ & $0.15 \pm 0.02$ & $\mathrm{k}$ \\
\hline & $0.468 \pm 0.023$ & $0.69 \pm 0.05$ & $83.1 \pm 2.6$ & $0.43 \pm 0.04$ & $\mathrm{c} 1$ \\
\hline & $0.085 \pm 0.004$ & $1.12 \pm 0.05$ & $66.1 \pm 2.7$ & $0.23 \pm 0.02$ & c2 \\
\hline & $0.093 \pm 0.005$ & $1.21 \pm 0.06$ & $29.2 \pm 2.7$ & $0.87 \pm 0.09$ & c3 \\
\hline & $0.042 \pm 0.002$ & $4.07 \pm 0.06$ & $62.3 \pm 2.8$ & $1.42 \pm 0.14$ & $\mathrm{c} 5$ \\
\hline \multirow[t]{5}{*}{1996 Mar. 22} & $0.478 \pm 0.024$ & $0.00 \pm 0.00$ & $0.0 \pm 0.0$ & $0.15 \pm 0.02$ & $\mathrm{k}$ \\
\hline & $0.370 \pm 0.019$ & $0.71 \pm 0.05$ & $83.9 \pm 2.6$ & $0.44 \pm 0.04$ & $\mathrm{c} 1$ \\
\hline & $0.109 \pm 0.005$ & $1.07 \pm 0.05$ & $67.7 \pm 2.7$ & $0.28 \pm 0.03$ & c2 \\
\hline & $0.090 \pm 0.006$ & $1.44 \pm 0.07$ & $33.8 \pm 3.2$ & $0.99 \pm 0.10$ & c3 \\
\hline & $0.033 \pm 0.002$ & $4.41 \pm 0.06$ & $63.2 \pm 2.6$ & $1.34 \pm 0.13$ & $c 5$ \\
\hline \multirow[t]{4}{*}{1996 May 27} & $0.446 \pm 0.022$ & $0.00 \pm 0.00$ & $0.0 \pm 0.0$ & $0.00 \pm 0.10$ & $\mathrm{k}$ \\
\hline & $0.408 \pm 0.020$ & $0.77 \pm 0.05$ & $81.8 \pm 3.2$ & $0.38 \pm 0.04$ & $\mathrm{c} 1$ \\
\hline & $0.081 \pm 0.010$ & $1.24 \pm 0.08$ & $54.6 \pm 3.5$ & $0.48 \pm 0.05$ & $\mathrm{c} 2$ \\
\hline & $0.055 \pm 0.006$ & $1.42 \pm 0.10$ & $23.3 \pm 3.5$ & $0.73 \pm 0.07$ & c3 \\
\hline \multirow[t]{7}{*}{1996 Jul. 27} & $0.402 \pm 0.020$ & $0.00 \pm 0.00$ & $0.0 \pm 0.0$ & $0.11 \pm 0.10$ & $\mathrm{k}$ \\
\hline & $0.395 \pm 0.020$ & $0.79 \pm 0.05$ & $80.8 \pm 2.7$ & $0.48 \pm 0.05$ & $\mathrm{c} 1$ \\
\hline & $0.049 \pm 0.003$ & $1.23 \pm 0.05$ & $55.7 \pm 2.8$ & $0.35 \pm 0.04$ & $\mathrm{c} 2$ \\
\hline & $0.058 \pm 0.003$ & $1.34 \pm 0.08$ & $20.9 \pm 2.7$ & $0.62 \pm 0.06$ & c3 \\
\hline & $0.019 \pm 0.001$ & $2.51 \pm 0.06$ & $45.5 \pm 3.0$ & $1.12 \pm 0.11$ & $\mathrm{c} 4$ \\
\hline & $0.017 \pm 0.001$ & $4.14 \pm 0.05$ & $61.4 \pm 2.9$ & $0.52 \pm 0.05$ & $c 5$ \\
\hline & $0.012 \pm 0.001$ & $5.80 \pm 0.08$ & $65.4 \pm 2.7$ & $0.86 \pm 0.09$ & c6 \\
\hline \multirow[t]{4}{*}{1996 Sep. 27} & $0.379 \pm 0.019$ & $0.00 \pm 0.00$ & $0.0 \pm 0.0$ & $0.10 \pm 0.10$ & $\mathrm{k}$ \\
\hline & $0.391 \pm 0.020$ & $0.83 \pm 0.05$ & $76.9 \pm 2.4$ & $0.53 \pm 0.05$ & $\mathrm{c} 1$ \\
\hline & $0.090 \pm 0.005$ & $1.38 \pm 0.09$ & $29.7 \pm 2.5$ & $1.03 \pm 0.10$ & c3 \\
\hline & $0.026 \pm 0.001$ & $4.24 \pm 0.05$ & $63.8 \pm 2.4$ & $1.07 \pm 0.11$ & $\mathrm{c} 5$ \\
\hline \multirow[t]{5}{*}{1996 Dec. 06} & $0.327 \pm 0.016$ & $0.00 \pm 0.00$ & $0.0 \pm 0.0$ & $0.12 \pm 0.10$ & $\mathrm{k}$ \\
\hline & $0.329 \pm 0.016$ & $0.84 \pm 0.05$ & $75.0 \pm 2.4$ & $0.62 \pm 0.06$ & $\mathrm{c} 1$ \\
\hline & $0.033 \pm 0.002$ & $1.42 \pm 0.07$ & $17.1 \pm 2.5$ & $0.55 \pm 0.06$ & c3 \\
\hline & $0.017 \pm 0.001$ & $2.25 \pm 0.04$ & $35.2 \pm 2.4$ & $0.73 \pm 0.07$ & $\mathrm{c} 4$ \\
\hline & $0.021 \pm 0.001$ & $4.19 \pm 0.05$ & $62.7 \pm 2.4$ & $0.57 \pm 0.06$ & $\mathrm{c} 5$ \\
\hline \multirow[t]{5}{*}{1997 Mar 10} & $0.350 \pm 0.020$ & $0.00 \pm 0.00$ & $0.0 \pm 0.0$ & $0.22 \pm 0.02$ & $\mathrm{k}$ \\
\hline & $0.251 \pm 0.013$ & $0.89 \pm 0.13$ & $75.2 \pm 5.2$ & $0.66 \pm 0.07$ & $\mathrm{c} 1$ \\
\hline & $0.053 \pm 0.016$ & $1.55 \pm 0.10$ & $22.9 \pm 6.5$ & $0.76 \pm 0.08$ & c3 \\
\hline & $0.011 \pm 0.002$ & $2.29 \pm 0.43$ & $50.2 \pm 8.1$ & $0.39 \pm 0.04$ & $\mathrm{c} 4$ \\
\hline & $0.033 \pm 0.006$ & $3.96 \pm 0.23$ & $64.5 \pm 2.7$ & $1.43 \pm 0.14$ & $\mathrm{c} 5$ \\
\hline \multirow[t]{5}{*}{1998 Oct. 30} & $0.503 \pm 0.025$ & $0.00 \pm 0.00$ & $0.0 \pm 0.0$ & $0.17 \pm 0.02$ & $\mathrm{k}$ \\
\hline & $0.092 \pm 0.005$ & $0.77 \pm 0.06$ & $75.1 \pm 3.0$ & $0.82 \pm 0.08$ & c1 \\
\hline & $0.033 \pm 0.002$ & $1.83 \pm 0.05$ & $32.4 \pm 2.9$ & $1.03 \pm 0.10$ & c3 \\
\hline & $0.019 \pm 0.003$ & $2.89 \pm 0.06$ & $54.2 \pm 2.6$ & $0.90 \pm 0.09$ & $\mathrm{c} 4$ \\
\hline & $0.018 \pm 0.002$ & $3.93 \pm 0.10$ & $67.3 \pm 2.6$ & $1.01 \pm 0.10$ & c5 \\
\hline \multirow[t]{5}{*}{1999 Sep. 1} & $0.402 \pm 0.020$ & $0.00 \pm 0.00$ & $0.0 \pm 0.0$ & $0.17 \pm 0.02$ & $\mathrm{k}$ \\
\hline & $0.157 \pm 0.008$ & $0.32 \pm 0.06$ & $76.8 \pm 3.6$ & $0.19 \pm 0.02$ & $\mathrm{ca}$ \\
\hline & $0.031 \pm 0.006$ & $0.84 \pm 0.10$ & $84.7 \pm 3.4$ & $0.43 \pm 0.04$ & $\mathrm{c} 1$ \\
\hline & $0.040 \pm 0.002$ & $1.83 \pm 0.07$ & $40.3 \pm 3.3$ & $1.20 \pm 0.12$ & c3 \\
\hline & $0.027 \pm 0.001$ & $3.64 \pm 0.06$ & $63.5 \pm 3.0$ & $1.10 \pm 0.11$ & c5 \\
\hline \multirow[t]{3}{*}{2000 Jan. 11} & $0.502 \pm 0.025$ & $0.00 \pm 0.00$ & $0.0 \pm 0.0$ & $0.10 \pm 0.10$ & $\mathrm{k}$ \\
\hline & $0.141 \pm 0.007$ & $0.41 \pm 0.04$ & $77.1 \pm 2.6$ & $0.31 \pm 0.03$ & $\mathrm{ca}$ \\
\hline & $0.045 \pm 0.003$ & $0.79 \pm 0.06$ & $71.7 \pm 3.5$ & $0.52 \pm 0.05$ & $\mathrm{c} 1$ \\
\hline
\end{tabular}


A\&A 515, A105 (2010)

Table 1. continued

\begin{tabular}{|c|c|c|c|c|c|}
\hline Epoch & Flux-density [Jy] & Core sep. [mas] & Pos. a. [deg] & Maj. a. [mas] & Id. \\
\hline \multirow{7}{*}{2000 May 20} & $0.009 \pm 0.002$ & $1.69 \pm 0.06$ & $18.0 \pm 5.0$ & $0.50 \pm 0.05$ & $\mathrm{x}$ \\
\hline & $0.009 \pm 0.001$ & $2.22 \pm 0.06$ & $44.2 \pm 3.1$ & $0.54 \pm 0.05$ & $\mathrm{c} 3$ \\
\hline & $0.026 \pm 0.001$ & $3.55 \pm 0.04$ & $62.1 \pm 2.6$ & $1.06 \pm 0.11$ & $c 5$ \\
\hline & $0.558 \pm 0.028$ & $0.00 \pm 0.00$ & $0.0 \pm 0.0$ & $0.14 \pm 0.01$ & $\mathrm{k}$ \\
\hline & $0.147 \pm 0.008$ & $0.35 \pm 0.05$ & $78.6 \pm 2.5$ & $0.31 \pm 0.03$ & $\mathrm{ca}$ \\
\hline & $0.077 \pm 0.004$ & $0.88 \pm 0.05$ & $76.2 \pm 2.5$ & $0.51 \pm 0.05$ & $\mathrm{cb}$ \\
\hline & $0.009 \pm 0.001$ & $1.72 \pm 0.05$ & $28.5 \pm 2.5$ & $0.77 \pm 0.08$ & $\mathrm{x}$ \\
\hline \multirow{7}{*}{2001 Oct. 22} & $0.018 \pm 0.010$ & $2.34 \pm 0.16$ & $47.0 \pm 4.9$ & $0.98 \pm 0.10$ & $\mathrm{c} 3$ \\
\hline & $0.007 \pm 0.002$ & $3.16 \pm 0.05$ & $63.9 \pm 2.8$ & $0.50 \pm 0.05$ & $\mathrm{c} 4$ \\
\hline & $0.016 \pm 0.001$ & $3.99 \pm 0.05$ & $64.2 \pm 2.6$ & $0.98 \pm 0.10$ & $\mathrm{c} 5$ \\
\hline & $0.608 \pm 0.030$ & $0.00 \pm 0.00$ & $0.0 \pm 0.0$ & $0.19 \pm 0.02$ & $\mathrm{k}$ \\
\hline & $0.327 \pm 0.016$ & $0.41 \pm 0.05$ & $56.4 \pm 2.6$ & $0.26 \pm 0.03$ & $\mathrm{ca}$ \\
\hline & $0.195 \pm 0.013$ & $0.90 \pm 0.05$ & $68.9 \pm 2.7$ & $0.66 \pm 0.07$ & $\mathrm{cb}$ \\
\hline & $0.022 \pm 0.001$ & $2.15 \pm 0.05$ & $55.3 \pm 2.7$ & $1.21 \pm 0.12$ & $\mathrm{cc}$ \\
\hline \multirow{4}{*}{2001 Dec. 23} & $0.018 \pm 0.001$ & $3.65 \pm 0.05$ & $63.3 \pm 2.4$ & $1.09 \pm 0.11$ & $\mathrm{~cd}$ \\
\hline & $0.640 \pm 0.032$ & $0.00 \pm 0.00$ & $0.0 \pm 0.0$ & $0.18 \pm 0.10$ & $\mathrm{k}$ \\
\hline & $0.335 \pm 0.017$ & $0.46 \pm 0.05$ & $56.0 \pm 2.6$ & $0.25 \pm 0.03$ & $\mathrm{ca}$ \\
\hline & $0.251 \pm 0.013$ & $0.91 \pm 0.05$ & $67.7 \pm 2.9$ & $0.78 \pm 0.08$ & $\mathrm{cb}$ \\
\hline \multirow{5}{*}{2002 Nov. 23} & $0.015 \pm 0.001$ & $2.33 \pm 0.06$ & $55.0 \pm 2.8$ & $0.98 \pm 0.10$ & $\mathrm{cc}$ \\
\hline & $0.017 \pm 0.001$ & $3.52 \pm 0.05$ & $65.9 \pm 2.6$ & $1.26 \pm 0.13$ & $\mathrm{~cd}$ \\
\hline & $0.702 \pm 0.035$ & $0.00 \pm 0.00$ & $0.0 \pm 0.0$ & $0.09 \pm 0.10$ & $\mathrm{k}$ \\
\hline & $0.312 \pm 0.016$ & $0.33 \pm 0.05$ & $68.0 \pm 2.6$ & $0.19 \pm 0.02$ & $\mathrm{ca}$ \\
\hline & $0.108 \pm 0.005$ & $0.84 \pm 0.05$ & $53.2 \pm 2.7$ & $0.30 \pm 0.03$ & $\mathrm{cb}$ \\
\hline \multirow{5}{*}{2003 Aug. 28} & $0.214 \pm 0.011$ & $1.21 \pm 0.05$ & $63.9 \pm 2.6$ & $0.68 \pm 0.07$ & $\mathrm{x}$ \\
\hline & $0.065 \pm 0.004$ & $2.23 \pm 0.08$ & $62.6 \pm 3.4$ & $1.63 \pm 0.16$ & $\mathrm{cc}$ \\
\hline & $0.640 \pm 0.032$ & $0.00 \pm 0.00$ & $0.0 \pm 0.0$ & $0.16 \pm 0.02$ & $\mathrm{k}$ \\
\hline & $0.178 \pm 0.009$ & $0.52 \pm 0.05$ & $63.4 \pm 2.9$ & $0.31 \pm 0.03$ & $\mathrm{ca}$ \\
\hline & $0.224 \pm 0.030$ & $0.94 \pm 0.06$ & $55.8 \pm 2.9$ & $0.56 \pm 0.06$ & $\mathrm{cb}$ \\
\hline \multirow{3}{*}{2004 Aug. 9} & $0.169 \pm 0.027$ & $2.20 \pm 0.07$ & $60.9 \pm 2.9$ & $1.61 \pm 0.16$ & $\mathrm{cc}$ \\
\hline & $0.523 \pm 0.026$ & $0.00 \pm 0.00$ & $0.0 \pm 0.0$ & $0.17 \pm 0.02$ & $\mathrm{k}$ \\
\hline & $0.103 \pm 0.008$ & $0.52 \pm 0.06$ & $62.8 \pm 4.7$ & $0.22 \pm 0.02$ & $\mathrm{ca}$ \\
\hline \multirow{7}{*}{2004 Dec. 2} & $0.184 \pm 0.009$ & $1.27 \pm 0.08$ & $63.8 \pm 4.3$ & $0.71 \pm 0.07$ & $\mathrm{cb}$ \\
\hline & $0.101 \pm 0.008$ & $2.52 \pm 0.09$ & $52.4 \pm 3.2$ & $1.54 \pm 0.15$ & $\mathrm{cc}$ \\
\hline & $0.060 \pm 0.003$ & $3.33 \pm 0.06$ & $65.9 \pm 3.0$ & $0.95 \pm 0.10$ & $\mathrm{~cd}$ \\
\hline & $0.522 \pm 0.026$ & $0.00 \pm 0.00$ & $0.0 \pm 0.0$ & $0.17 \pm 0.02$ & $\mathrm{k}$ \\
\hline & $0.140 \pm 0.011$ & $0.58 \pm 0.07$ & $62.2 \pm 3.4$ & $0.32 \pm 0.03$ & $\mathrm{ca}$ \\
\hline & $0.115 \pm 0.012$ & $1.26 \pm 0.06$ & $62.7 \pm 2.6$ & $0.85 \pm 0.09$ & $\mathrm{cb}$ \\
\hline & $0.112 \pm 0.014$ & $2.52 \pm 0.06$ & $56.6 \pm 4.4$ & $1.44 \pm 0.14$ & $\mathrm{cc}$ \\
\hline \multirow{4}{*}{2006 Apr. 28} & $0.057 \pm 0.010$ & $3.46 \pm 0.09$ & $64.1 \pm 2.8$ & $0.97 \pm 0.10$ & $\mathrm{~cd}$ \\
\hline & $0.503 \pm 0.025$ & $0.00 \pm 0.00$ & $0.0 \pm 0.0$ & $0.19 \pm 0.02$ & $\mathrm{k}$ \\
\hline & $0.110 \pm 0.006$ & $0.49 \pm 0.05$ & $63.0 \pm 2.9$ & $0.36 \pm 0.04$ & $\mathrm{ca}$ \\
\hline & $0.153 \pm 0.008$ & $1.10 \pm 0.05$ & $60.7 \pm 2.8$ & $0.65 \pm 0.07$ & $\mathrm{cb}$ \\
\hline \multirow{6}{*}{2007 Mar. 2} & $0.026 \pm 0.001$ & $2.25 \pm 0.05$ & $51.7 \pm 2.8$ & $1.58 \pm 0.16$ & $\mathrm{cc}$ \\
\hline & $0.105 \pm 0.005$ & $3.41 \pm 0.05$ & $62.7 \pm 2.8$ & $1.30 \pm 0.13$ & $\mathrm{~cd}$ \\
\hline & $0.408 \pm 0.020$ & $0.00 \pm 0.00$ & $0.0 \pm 0.0$ & $0.17 \pm 0.02$ & $\mathrm{k}$ \\
\hline & $0.036 \pm 0.005$ & $0.55 \pm 0.06$ & $55.8 \pm 8.2$ & $0.04 \pm 0.10$ & $\mathrm{ca}$ \\
\hline & $0.141 \pm 0.016$ & $0.95 \pm 0.08$ & $67.0 \pm 2.9$ & $0.66 \pm 0.07$ & $\mathrm{cb}$ \\
\hline & $0.055 \pm 0.003$ & $2.26 \pm 0.06$ & $53.7 \pm 2.9$ & $1.11 \pm 0.11$ & $\mathrm{cc}$ \\
\hline \multirow{4}{*}{2007 Aug. 9} & $0.074 \pm 0.004$ & $3.65 \pm 0.05$ & $64.2 \pm 2.9$ & $1.39 \pm 0.14$ & $\mathrm{~cd}$ \\
\hline & $0.470 \pm 0.024$ & $0.00 \pm 0.00$ & $0.0 \pm 0.0$ & $0.20 \pm 0.02$ & $\mathrm{k}$ \\
\hline & $0.043 \pm 0.003$ & $0.46 \pm 0.06$ & $77.3 \pm 3.8$ & $0.30 \pm 0.03$ & $\mathrm{ca}$ \\
\hline & $0.094 \pm 0.014$ & $1.06 \pm 0.05$ & $61.9 \pm 4.3$ & $0.58 \pm 0.06$ & $\mathrm{cb}$ \\
\hline \multirow{7}{*}{2008 Nov. 26} & $0.064 \pm 0.013$ & $2.32 \pm 0.13$ & $56.8 \pm 5.3$ & $1.48 \pm 0.15$ & $\mathrm{cc}$ \\
\hline & $0.072 \pm 0.006$ & $3.54 \pm 0.08$ & $63.4 \pm 3.1$ & $1.37 \pm 0.14$ & $\mathrm{~cd}$ \\
\hline & $0.327 \pm 0.016$ & $0.00 \pm 0.00$ & $0.0 \pm 0.0$ & $0.14 \pm 0.10$ & $\mathrm{k}$ \\
\hline & $0.122 \pm 0.006$ & $0.41 \pm 0.05$ & $76.0 \pm 3.0$ & $0.29 \pm 0.03$ & $\mathrm{ca}$ \\
\hline & $0.072 \pm 0.004$ & $0.97 \pm 0.05$ & $67.8 \pm 2.9$ & $0.53 \pm 0.05$ & $\mathrm{cb}$ \\
\hline & $0.039 \pm 0.002$ & $2.37 \pm 0.06$ & $56.2 \pm 2.9$ & $1.60 \pm 0.16$ & $\mathrm{cc}$ \\
\hline & $0.075 \pm 0.004$ & $3.65 \pm 0.05$ & $63.7 \pm 2.9$ & $1.42 \pm 0.14$ & $\mathrm{~cd}$ \\
\hline
\end{tabular}


observation, the flux-density of each fitted component and its separation from the core, the corresponding position angle, and the component size.

\section{Results}

Figures 1 and 2 show the images derived by us, with the modelfit components superimposed. It is evident that the jet's morphology undergoes a drastic change during the time span covered by our observations. In Fig. 1 the jet shows the twice bent structure - the staircase mode, whereas in Fig. 2 the jet structure is seen to have become straight. Figure 3 a shows the variation of core separations between the epochs 1995.27 and 2008.91 for the different components. Striking variations in the jet kinematics are seen during the period 1995-2000.4. Four jet components clearly show outward directed motion with apparent superluminal speeds, while one component shows an inward motion (directed towards the core) also with an apparent superluminal speed. The apparent speeds derived from linear regressions to the component positions are given in Table 2. From 2001.8 on, only four components can be identified in the VLBI jet, and the jet components are found to be stationary (Figs. 1 and 2).

Figure $3 \mathrm{~b}$ presents the position angle (relative to the core) at different epochs for each component. Again we find a significant change to have occured between 2000.4 and 2001.8. Between 1995 and 2000 the position angle distribution is quite broad with values ranging from $\sim 10^{\circ}-100^{\circ}$, which amounts to a wide spread of over $\sim 90^{\circ}$. After 2000.4 , the position angle distribution is seen to become much narrower, with a total spread of just about $20^{\circ}$ around a mean value of $\sim 65^{\circ}$.

In Fig. $3 c$ the relation between the core separation and position angle is plotted for each of the 23 epochs. This plot more clearly depicts the two contrasting trends present in the VLBI images. Thus, a different pattern is observed for the epochs before 2000.4 and after 2000.4. Prior to 2000.4 all the jet ridge lines show a similar form, with a clear minimum at a core separation of $\sim 1.5$ mas, where the position angle drops to $\sim 20^{\circ}$. After 2000.4, the form of the jet ridge lines becomes almost linear.

Thus, both the jet morphology and kinematics are found to change remarkably between 1995 and 2009, as summarized below:

1. In the staircase jet mode five radio knots/components move with apparent superluminal speeds either away from the core (four components), or towards it (one component). The position angle distribution is broad $\left(\sim 90^{\circ}\right)$. The jet ridge lines for all epochs before 2000.4 are similar, with a clear minimum in position angle.

2. In the straight jet mode, separations of all four VLBI components from the core remain essentially unchanged; their apparent speeds are sub-luminal. The position angle distribution is narrow $\left(\sim 20^{\circ}\right)$.

In Fig. 3d we present the same information as in Fig. 3c, but for selected epochs only: the two epochs before the mode change and the first three epochs following the mode change (2001.81, 2001.98 and 2002.90). Here the different jet ridge lines for the two modes are seen more clearly. Figure $3 e$ displays the evolution of flux-densities of the individual components and the core; again significant changes are observed.

\subsection{Morphological transitions and flux-density variability}

The BL Lac $0735+178$ is known for its pronounced flux variability in both optical and radio bands (Ciprini et al. 2007;
Goyal et al. 2009). The origin of this variability, however, is still debated. To check for a possible correlation between the optical/radio flux-density variability and the morphological transitions witnessed in the VLBI observations, we show the light curves (Figs. 3f, 4) with the epochs of the observed mode transitions indicated by pairs of grey and black lines, respectively.

\subsubsection{Radio flux variability}

In Fig. $3 f$ the intergrated flux-densities at $4.8,8.0$, and $14.5 \mathrm{GHz}$ are plotted, monitored under the University of Michigan programme (UMRAO). The grey stripes denote the two most recent mode transition events. Based on the VLBI images reported here and in the literature (see above), one can deduce that the first of these mode transitions (straight to staircase) occured sometime between mid-1992 and mid-1995, and hence its time-scale must be less than three years. Likewise, the latest mode transition (staircase to straight) we report in this paper took less than two years. The time interval between the two transitions is $\sim 7.5$ years.

In Fig. 4 we show a plot adopted from Goyal et al. (2009) and indicate in it all three known mode transitions within pairs of vertical black lines. The 2001 transition occured shortly before a clear radio flare. The 1994 transition occured shortly before a clear modest radio flare. The 1983 mode transition cannot be related to a significant radio flare. While the corelation between the times of the transititons and the optical flaring is convincing, the case is not so clear with regard to a possible correlation with radio flares. It seems that for the last two transitions the radio flares are delayed with regard to the optical flare, which could be explained by propagation effects within the source. Unfortunately only one VLBI observation - the observation by Gabuzda et al. (1994) from 1990.47 - is available for the time of the most prominent radio flaring around 1990. This would help to understand the relation between the radio flaring and the morphological changes. A simultaneous monitoring of the radio and optical flux in combination with multi radio frequency VLBI observation is required for a better understanding of the emission processes.

\subsubsection{Correlation with optical flux variability}

In Fig. 4a, b we reproduce the optical light curves from Goyal et al. (2009), on which we have marked the three known mode transitions mentioned above. Both staircase to straight mode changes clearly take place near the brightness maxima. The optical flares coincident with these two transitions are more prominent compared to the middle flare coincident with the opposite mode transition. We thus find that a strong correlation exists between the structural change in the parsec-scale jet and the optical light curve. To our knowledge, such a correlation has not been pointed out earlier for any jet, and this underscores the importance of long-term, coordinated VLBI and optical monitoring of blazars.

The origin of this conspicuous correlation in this source needs to be investigated in greater detail. This correlation could be explained by a small change in the direction to the line of sight. This would change the amplification of intrisinc jet bending and also allow for a more direct view into the inner regions of the AGN. This "geometric" explanation should be explored further. While more subtle interpretations are possible, a simple possibility to understand this correlated radio-optical behavior is that the optical flare manifests an impulse by which a large 
A\&A 515, A105 (2010)

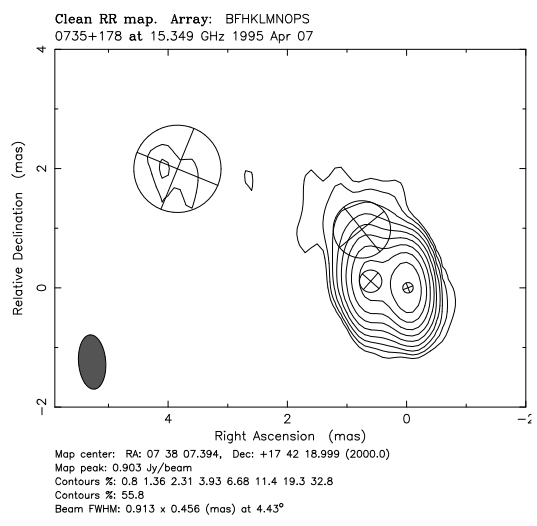

(a)

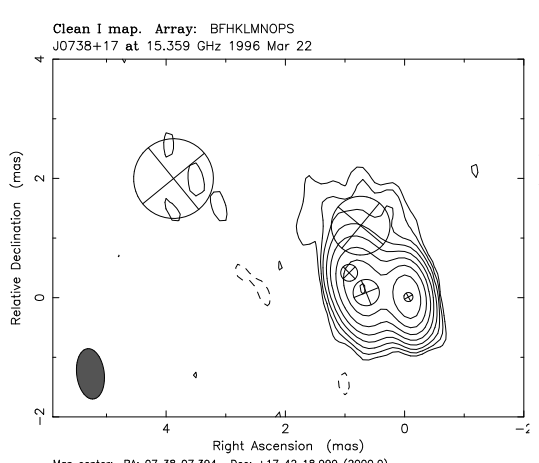

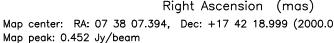

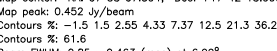

(d)

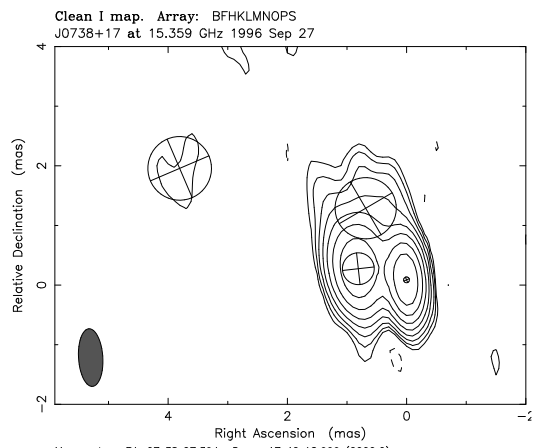

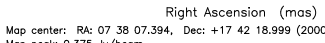

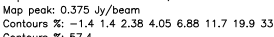

Sem

(g)

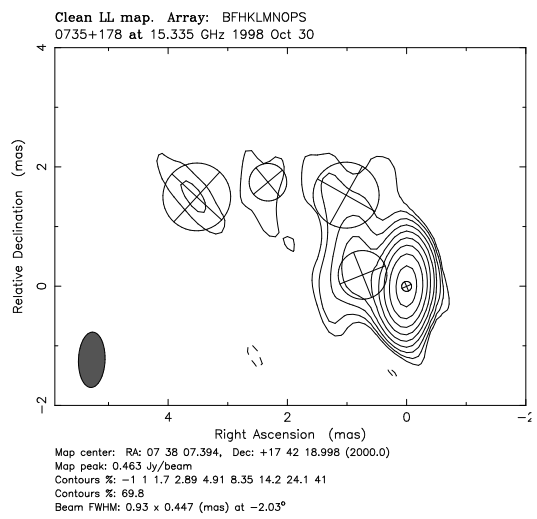

(j)
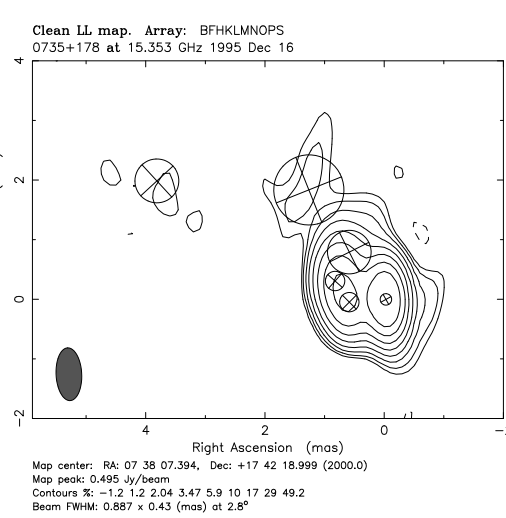

(b)

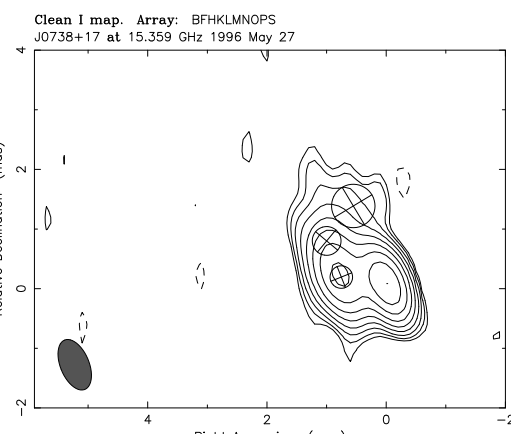

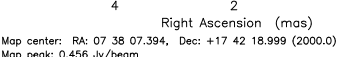

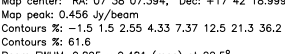

(e)
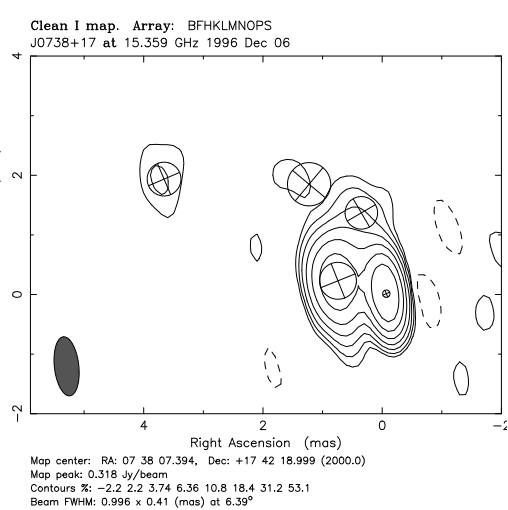

(h)

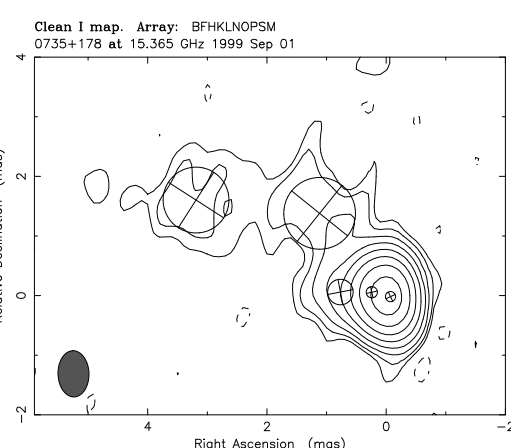

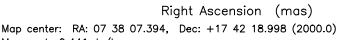

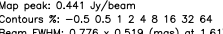

(k)

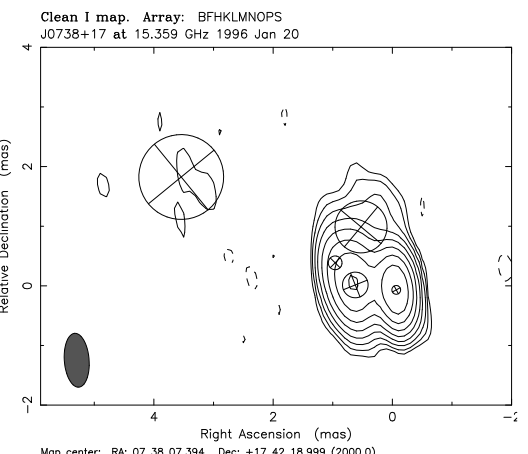

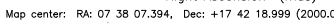

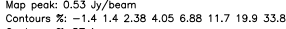

(c)

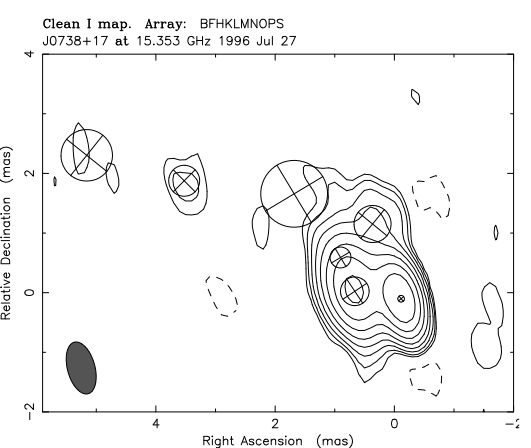

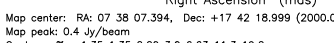

Conteurs

(f)

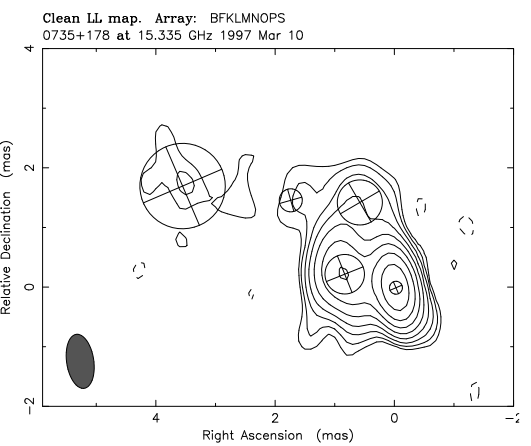

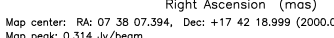

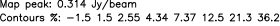

(i)

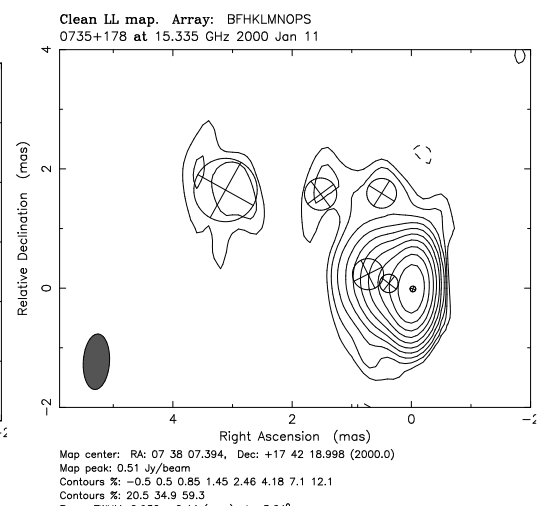

(1)

Fig. 1. The VLBI images superimposed with model-fitting results are shown for all epochs between 1995.27 and 2000.03. 
S. Britzen et al.: Understanding BL Lacertae objects

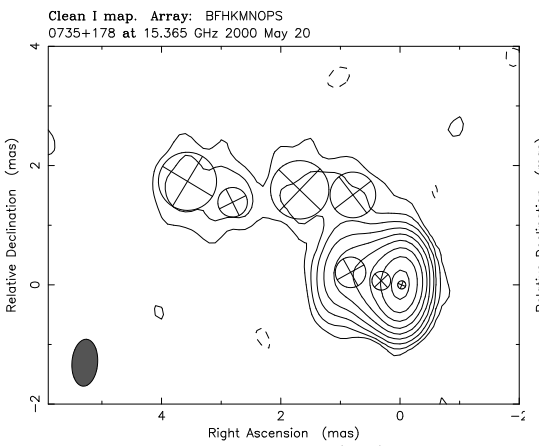

Right Ascension (mas)
Map center: RA: 0738 07.394, Decs: $+174218,998$ (2000)

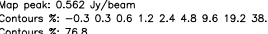

(a)

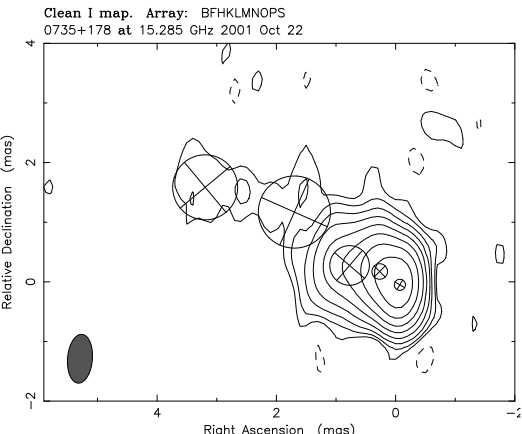

Hap center: R4. 0738 07.394, Decis +1742 19.998 (2000.0)

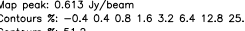

(b)

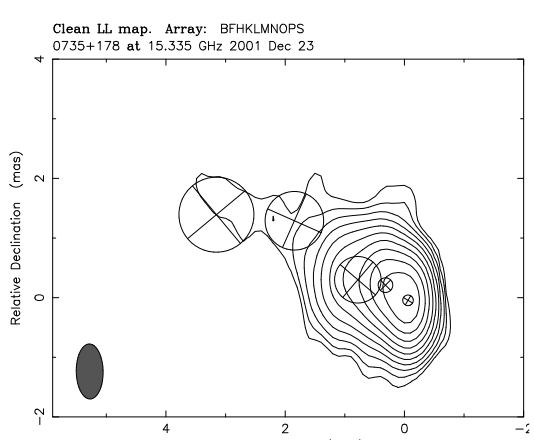

Right Ascension (mas)
Map center: FA: 0738 07.394, Des: +174218.998 (2000.0)

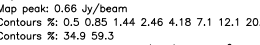

(c)

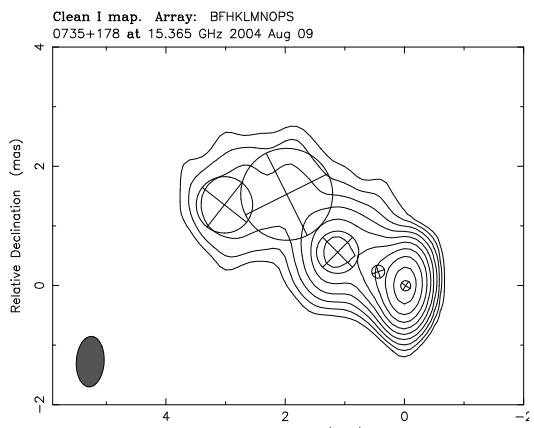

$\begin{aligned} & \text { Right Ascension (mas) } \\ & \text { Hap center: RA: } 0738 \text { 07.394., Dec: }+174218.998 \text { (2000.0) }\end{aligned}$

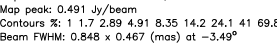

(f)

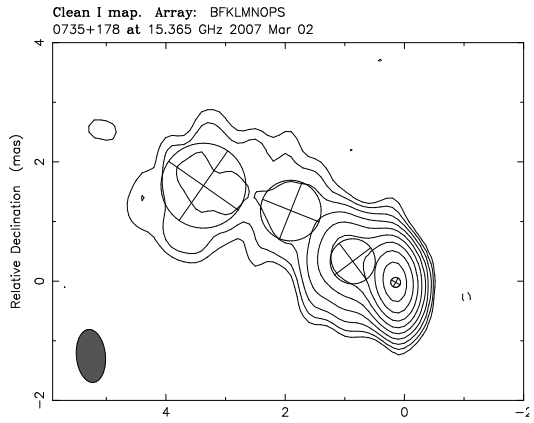

$\begin{aligned} & \text { Right Ascension (mas) } \\ & \text { Hap center: F4: } 0738 \text { 07.394, Dec: }+174218.998 \text { (2000.0) }\end{aligned}$

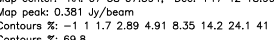

(i)

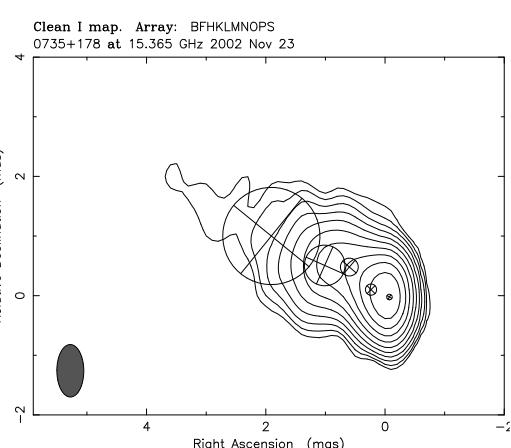

Right Ascension (mas)
Map center: RA: 0738 07.394. Deaci +1742 18.996 (20000.0)

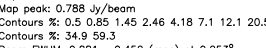

(d)

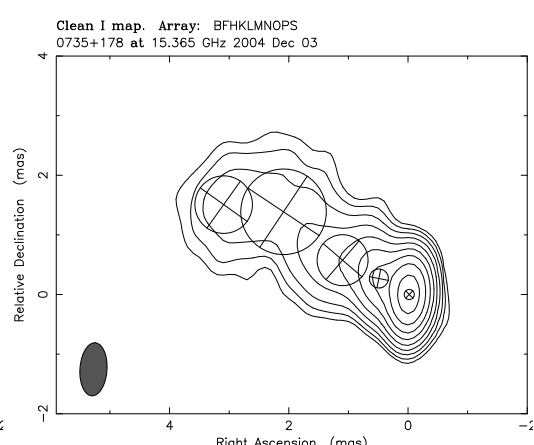

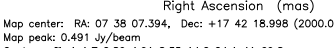

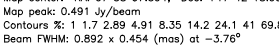

(g)

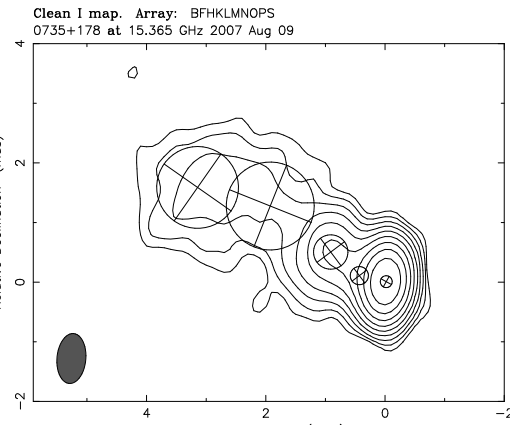

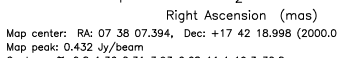

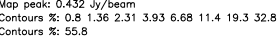

(j)

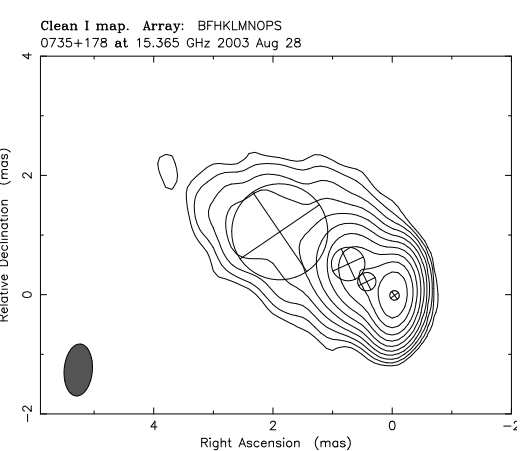

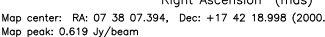

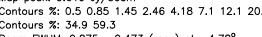

(e)

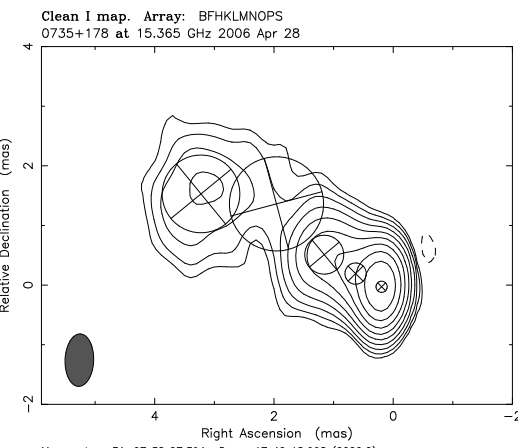

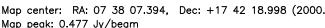

(1)

(h)

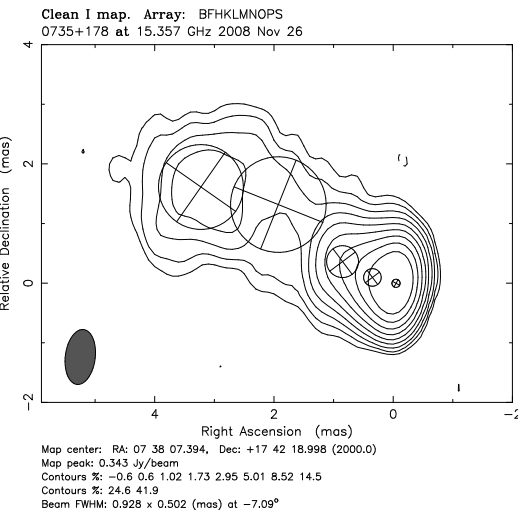

(k)

Fig. 2. The VLBI images superimposed with model-fitting results are shown for all epochs between 2001.98 and 2008.91. 


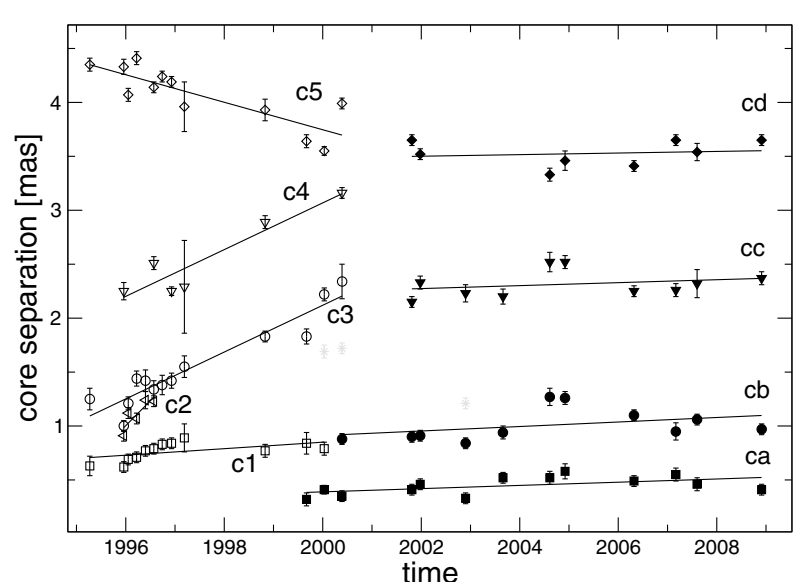

(a)

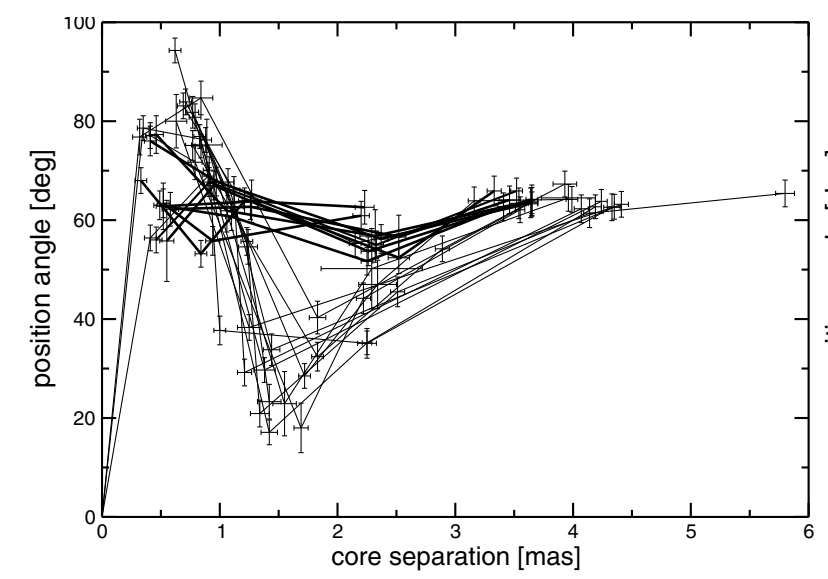

(c)

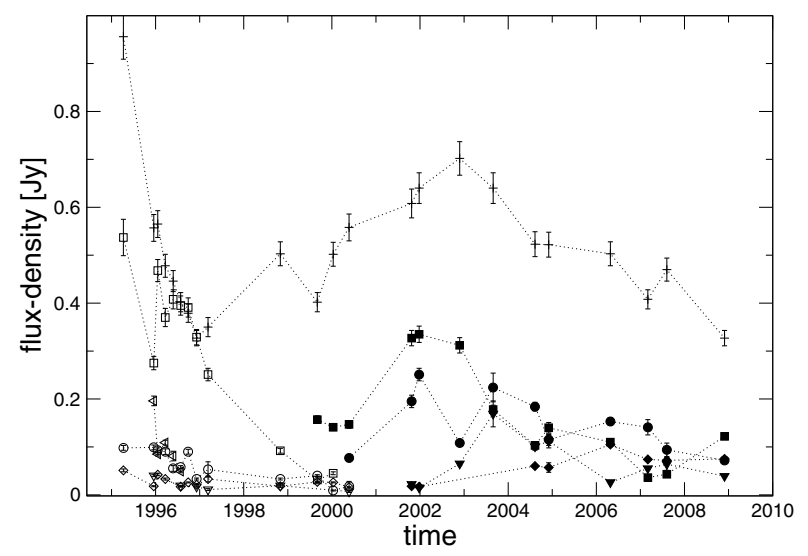

(e)

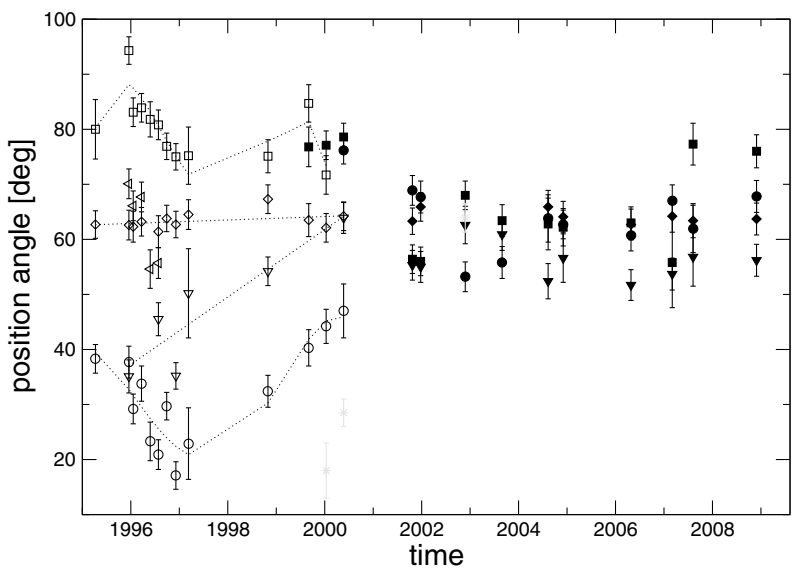

(b)

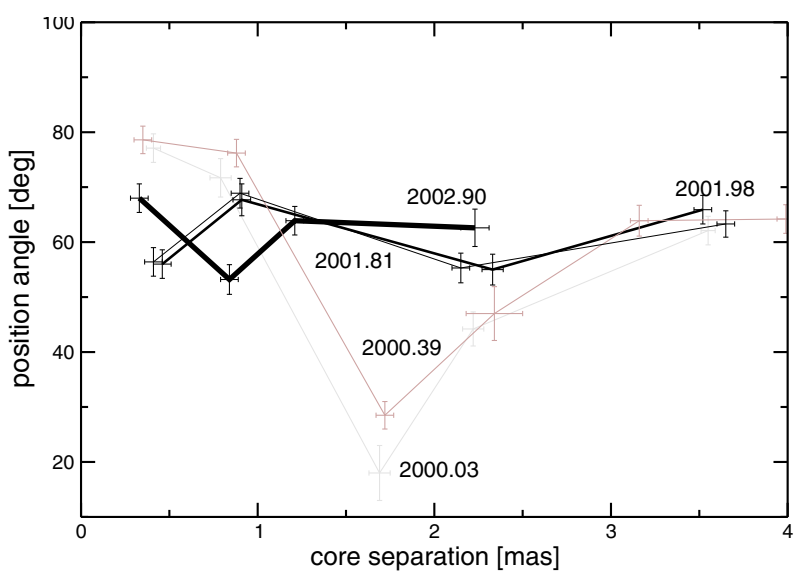

(d)

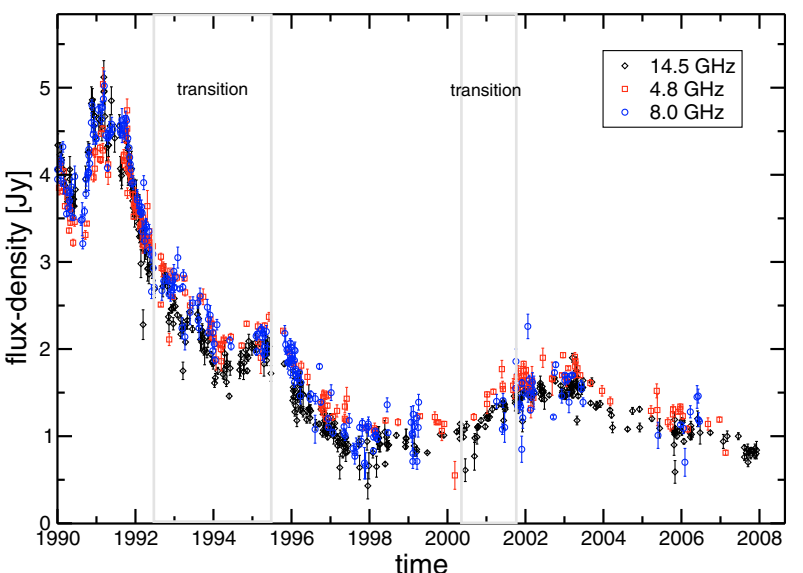

(f)

Fig. 3. a) Core separation of each VLBI component as function of time for both time intervals described in the text. Grey symbols represent weak components that could not be identified through the epochs. b) Position angle of each component as function of time, for both time intervals. c) The jet ridge-line for all the epochs observed. d) Three representative jet ridge-lines selected from the two time intervals, for comparison. e) Fluxdensity evolution of the individual components and the core (upper data points). f) Radio flux-density light-curves from the Michigan monitoring program (UMRAO). Indicated are the two transition phases where the mode changes (i.e., morphological transitions) occurred (Sect. 3).

amount of kinetic power (momentum flux) is injected by the central engine into the nuclear jet, as a result of which the jet gets stretched into a linear shape. This explanation is related to the question of higher stability of powerful jets and the collimation process of jets in general (e.g., Nakamura \& Meier 2009). The observed time lag between the optical flare and the disappearance of the jet's kink could in fact be quite small $(<1 \mathrm{yr})$, since the jet flow in blazars is directed close to the line-of-sight, which is also the direction of propagation of the optical signal recorded. An alternative scenario is presented in Sect. 4.4. Either of these two possible models will also be required to explain the disappearance of superluminal motion following the mode transition to a straight jet.

The proposed energetic impulse that straightens the kinky jet could originate from the core. Assuming that $0735+178$ is a helical jet, whose components are therefore seen at different 
Table 2. Proper motion and apparent speeds of the individual jet components in $0735+178$ (Sect. 3).

\begin{tabular}{lccc}
\hline \hline Mode & Component & $\mu[\mathrm{mas} / \mathrm{year}]$ & $\beta_{\mathrm{app}}[c]$ \\
\hline staircase & $\mathrm{c} 1$ & $0.03 \pm 0.01$ & $0.77 \pm 0.26$ \\
& $\mathrm{c} 2$ & $0.46 \pm 0.16$ & $11.87 \pm 4.16$ \\
& $\mathrm{c} 3$ & $0.22 \pm 0.02$ & $5.68 \pm 0.52$ \\
& $\mathrm{c} 4$ & $0.22 \pm 0.04$ & $5.68 \pm 1.03$ \\
& $\mathrm{c} 5$ & $-0.13 \pm 0.03$ & $-3.36 \pm 0.77$ \\
\hline straight & $\mathrm{ca}$ & $0.01 \pm 0.01$ & $0.26 \pm 0.26$ \\
& $\mathrm{cb}$ & $0.02 \pm 0.02$ & $0.52 \pm 0.52$ \\
& $\mathrm{cc}$ & $0.01 \pm 0.02$ & $0.26 \pm 0.52$ \\
& $\mathrm{~cd}$ & $0.01 \pm 0.02$ & $0.26 \pm 0.52$ \\
\hline
\end{tabular}

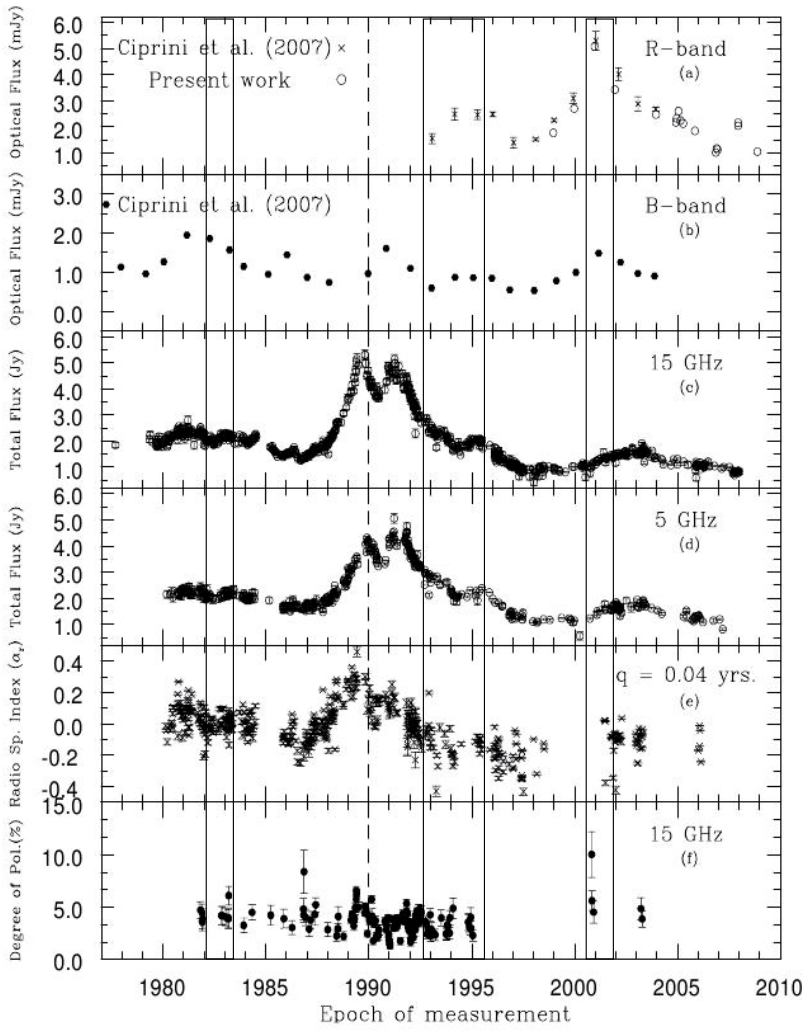

Fig. 4. The diagram, reproduced from Goyal et al. (2009), shows the multiple light-curves of PKS $0735+178$. Also displayed are the $R$-band light curve a), the $B$-Band light curve b), the UMRAO light curves at 15 and $5 \mathrm{GHz}$ c) \& d) and the run of the radio spectral index e). The plot f) shows the variation of the percentage (linear) polarization at $15 \mathrm{GHz}$. The two pairs of vertical lines bracket the structural transition phases discussed in the text.

viewing angles at the times of different mode transitions, then this could explain the connection to the radio flares as well. The radio flare would then not be caused by the ejection of a new component - which is usually assumed - but by the Doppler factor changing with the viewing angle.

\section{Discussion}

\subsection{Morphological mode transitions}

PKS $0735+178$ showed a kinky jet trajectory in the VLBI observations in December 1981 and a straight jet trajectory in June
1983. Therefter, sometime between mid-1992 and mid-1995, the morphology changed back to kinky with two sharp bends of $\sim 90^{\circ}$ (staircase mode), within $2 \mathrm{~ms}$ of the core. Between 2000.4 and 2001.8 the jet trajectory changed once again to the straight mode, as found in the present work. Thus, three clear mode changes of the pc-scale trajectory of this jet were observed. The time gaps between these transitions are about 11.3 years and 7.2 years. A simple scenario for the mode transition from kinky to straight jet was outlined in the previous section, taking a clue from the observed coincidences of the morphological transition events (kinky to straight jet) with the optical flares of this blazar. Below we present a kinematic model for the latest mode transition revealed by our analysis of the VLBI observations taken at 23 epochs between 1995 to 2009 (Sect. 4.4).

\subsection{Jet kinematics}

During the staircase mode, outward and inward apparent superluminal motion is observed. During the straight mode, the separations of various knots/components from the core remained essentially unchanged. Whether these components are the same or different, cannot be decided with absolute certainty. However, should the mode transition be caused by a changing viewing angle - a possibility argued below - the components could be the same.

A similar phenomenon - rather stationary components relative to the core, but moving perpendicular to the jet ridge line - was studied by us in the two prominent BL Lac objects, $1803+784$ (Britzen et al. 2009) and 0716+714 (Britzen et al. 2009). Over a time of $\sim 20$ years we do find rather slow apparent motion or stationarity for nearly all of the components in the pc-scale jet of $1803+784$. However, the components are seen to move significantly in position angle measured from the core. The entire ridge line of the jet thus evolves in the transverse direction, changing from a straight line to an almost sinusoidal form and back again to a straight line. These significant jet ridge-line changes occur on a timescale of $\sim 8$ years. A similar phenomenon was studied by us in the highly variable BL Lac $0716+714$. Here again we find rather stationary components that do, however, show motion in position angle and, once again, the jet's ridge line evolves. We expect that the stationary components can be explained in terms of a geometric origin. In the past, various mechanisms were proposed to explain slow or subluminal apparent motions. Smaller apparent speeds can be obtained by a flux-weighted integration of the apparent motions over the entire jet width (Gopal-Krishna et al. 2004). For a brief review we refer to Britzen et al. (2008) and references therein.

Different studies have reached opposite conclusions on whether BL Lac objects typically have slow or fast jet components (e.g., Gabuzda et al. 1994; Wehrle et al. 1992; Ghisellini et al. 1993; Jorstad et al. 2001). The CJF (Caltech-Jodrell Bank Flat-Spectrum) survey of 293 AGN (Britzen et al. 2008) shows some tendency for smaller apparent velocities of BL Lac objects compared to quasars. However, the small size of the BL Lac sample handicaps the comparison.

\subsection{A "hybrid" between quasars and BL Lac objects?}

As noted in the previous subsection, there has been an ongoing debate in recent years whether BL Lac objects and Quasars reveal similar kinematic properties or whether the apparent speeds differ significantly. According to the general picture of the AGN paradigm, fast apparent speeds with values up to $\sim 30 c$ can be 


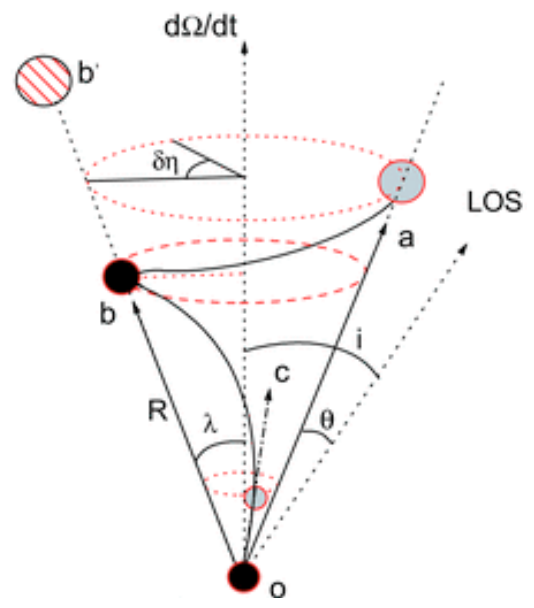

Fig. 5. This figure is reproduced from Gong (2008) and shows the difference between ballistic and non-ballistic motion. The model of ballistic motion plus precession is represented by the solid spiral connecting the features a), b), and c). Denoted by the dashed ellipse through feature b) is the non-ballistic model which precesses with a constant distance to the core.

Table 3. The parameters applied to fit the data of $0735+178$ in the nonballistic model.

\begin{tabular}{cccccc}
\hline \hline$\dot{\Omega}$ & $i$ & $\xi$ & $\lambda_{1}$ & $\lambda_{2}$ & $\lambda_{3}$ \\
15.0 & 9.8 & 225.6 & 3.5 & 3.0 & 4.6 \\
\hline$\eta_{1}$ & $\eta_{2}$ & $\eta_{3}$ & $R_{1}$ & $R_{2}$ & $R_{3}$ \\
165.2 & 21.7 & -53.0 & 14.0 & 11.1 & 4.5 \\
\hline
\end{tabular}

observed (e.g., Piner et al. 2006) and in almost all cases the motion is directed away from the assumed stationary core. This is in contrast to what we find in a growing number of BL Lac objects. Based on our analysis of $0735+178$ presented here and the detailed studies of $1803+784$ and $0716+714$, it is possible that the kinematic properties are significanlty different. $0735+178$ is a "hybrid" with regard to the mode changes and the accompanying kinematics - it shows "quasar"-like fast apparent motions at certain periods of time and then again "slow" motion (as we find in BL Lac objects) at other times. Thus this source switches between the different states that we tend to associate with quasars or BL Lac objects. Due to this atypical behavior, $0735+178$ can help us to understand the difference between these two types of AGN. It is often assumed that the basic difference between these two classes is based on a different line of sight to the object, with the BL Lacs being seen under the smallest angle to the line of sight (Unified Schemes). Within this scheme, it is then quite conceivable that the mode changes are caused by small changes in the angle to the line of sight, e.g., the jet bends slightly towards or away from us. Undoubtedly, $0735+178$ is an extreme object since these changes of the angle can not be very considerable in the source frame. Thus, $0735+178$ might already be oriented in a critical angle towards us. This would explain why this is the only source so far where these mode fluctutations have been observed.

\subsection{Helix or pressure gradients in the external medium?}

Gong (2008) proposed a non-ballistic model (see Fig. 5), in which a continuous jet produces discrete hotspots via the interaction of the relativistic outflow with the ambient medium. Thereby significant bulk energy of the jet is transferred to the

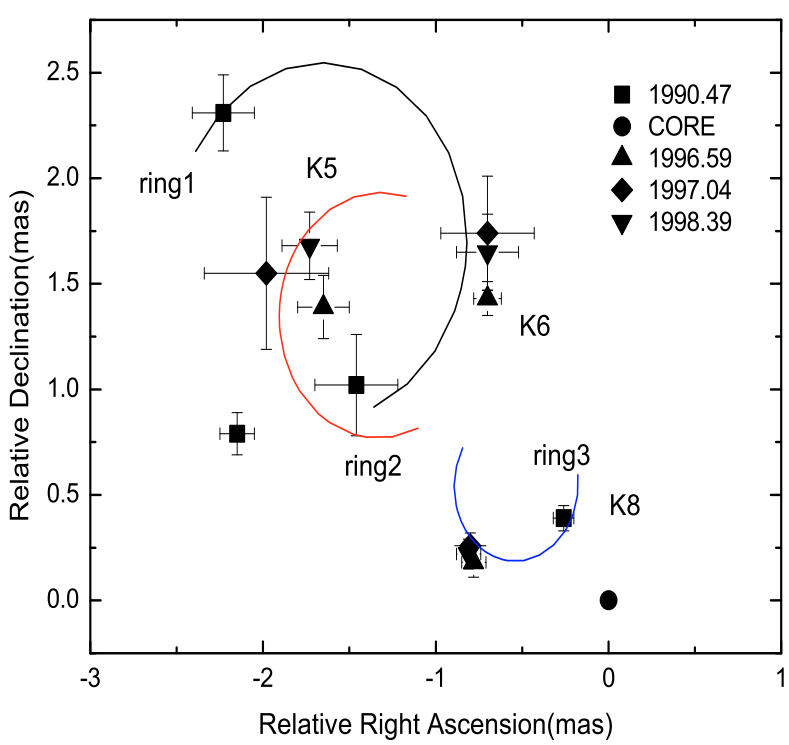

Fig. 6. The observations of the BL Lac object $0735+178$ at $8.4 \mathrm{GHz}$ from Gómez et al. (2001) as well as the curves fitted to the data.

swept up material. The precession of the jet axis results in the motion of hotspots at different directions along the precession cone, which resembles a hotspot moving along a ring-like trajectory. Significant bulk energy loss may occur repeatedly for a continuous jet with a fixed axis, leading to several distinct hotspots at different distances from the core. The precession of such a continuous jet results, on the other hand, in the motion of hotspot at different rings and with different precession phases (a detailed analysis is in preparation).

Under this scenario, the rectangular structure is just a state of the motion of these hotspots in different rings, which can appear and disappear depending on the precession phases of these hotspots and their projections on the sky plane. Thus, conceivably, the non-ballistic model of Gong (2008) can provide a fairly simple explanation for the appearance of the staircase mode.

Figure 6 shows the observation of the BL Lac object $0735+178$ at $8.4 \mathrm{GHZ}$ by Gómez et al. (2001) as well as the curves fitted to the data. The inner components K8, K6 and K5 - as defined in Gómez et al. (2001) - are fitted within the nonballistic model (Gong 2008) by three solid curves (corresponding to ring 1 , ring 2 and ring 3 , respectively) at different distances to the core. The evolution of the three curves is clockwise, all of them start in 1988.47 and end in 2002.97.

Under the simplest treatment, the non-ballistic model just gives the same opening angle for the precession cone for the three rings, $\lambda_{1}=\lambda_{2}=\lambda_{3}$. However, in this case the morphology of PKS $0735+178$ cannot be fitted well.

Thanks to the scenario of the pressure gradients in the external medium through which the jet propagates (Gómez et al. 2001), it is reasonable to assume that $\lambda_{1}, \lambda_{2}, \lambda_{3}$ can deviate from each other by a few degrees. Under these circumstances, the evolution of the staircase mode can be well fitted, as shown in Fig. 6. The input parameters to this model are given in Table 3.

The precession speed, $\dot{\Omega}$, is in units of $\mathrm{deg} / \mathrm{yr}$, the distance of a hotspot to the core, $R_{i}$, is in units of mas, and all other parameters are in units of degree. $i$ is the inclination angle between the jet's rotation axis and the line of sight; $\xi$ is the coordinate transformation angle; $\lambda_{i}$ is the opening angle of the precession cone; $\eta_{i}$ is the initial phase. 

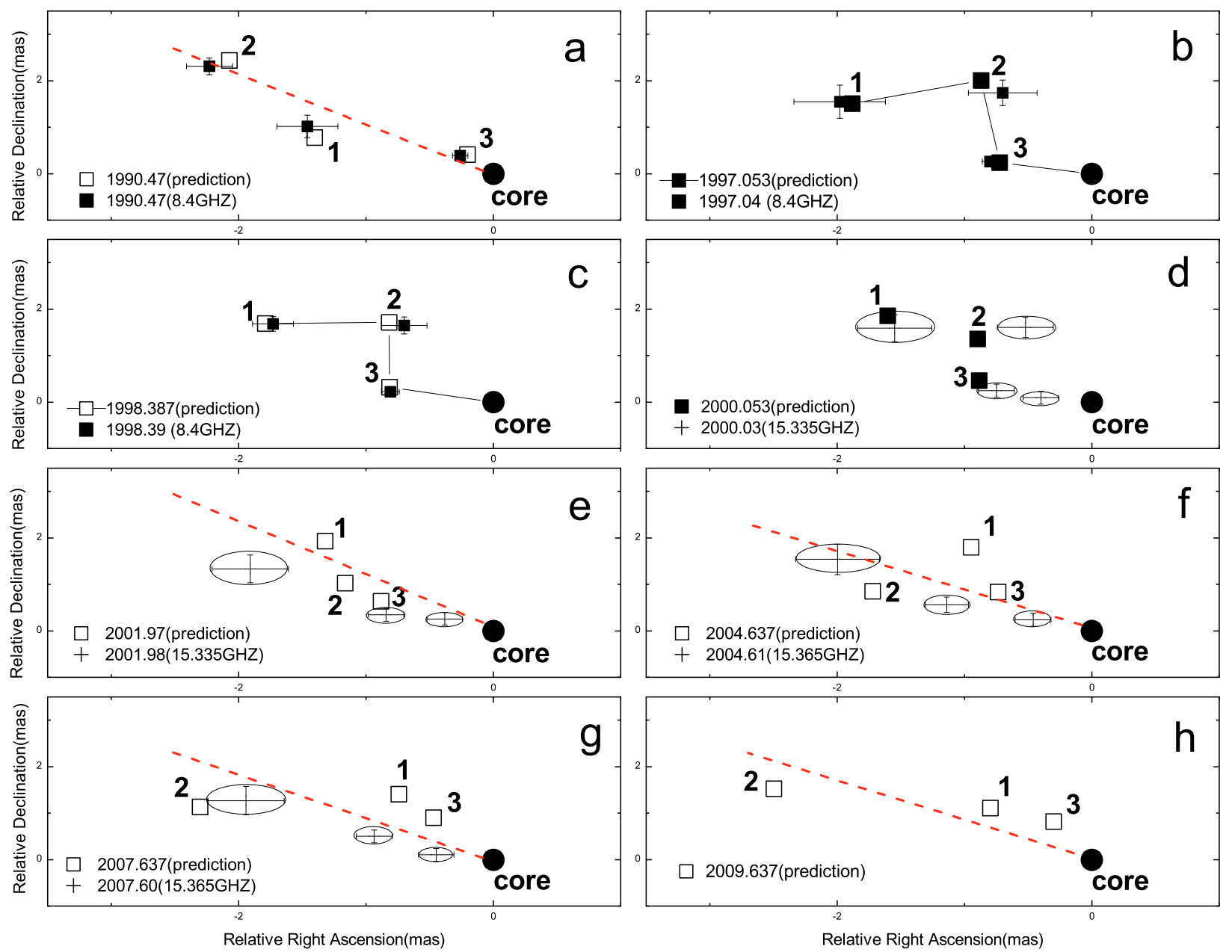

Fig. 7. The observed morphology of $0735+178$ is indicated by filled squares and ellipses, corresponding to the VLBI observations at 8.4 GHz (1990-1998) and at $15 \mathrm{GHz}$ (2000-2007). The evolution of the trajectory of hotspots (hollowed square) from 1990 to 2009 is reproduced in terms of the non-ballistic model.

Although Fig. 7 shows the fit to the $8.4 \mathrm{GHz}$ observations of Gómez et al. (2001), the theoretical trajectory (Fig. 7h can be extended to up to 2009.63. By doing this, the disappearance of the staircase observed at $15.335 \mathrm{GHz}$ and $15.365 \mathrm{GHz}$ (symbolized by the ellipses) at the end of 2001 can also be explained as shown in Fig. 7. This figure shows the evolution of the jet ridge line based on the observed morphology (filled filled squares and ellipses) and the model (open squares). For the time of 2009.637 the model prediction is shown in the last panel of this figure. This figure distinctly shows the different morphological phases of the jet ridge line as they are observed and how well they can be reproduced within the model.

Consequently, the staircase mode occurs when the hotspot precesses to certain phases so that the projected appearance is rectangular. Likewise, the staircase mode disappears when the hotspot precesses to other phases where the projection deviates from the rectangular image. Obviously, the precession of a hotspot varies the angle of the jet axis from the line of sight. This leads to a varying Doppler boosting, which explains the observed radio flux-density changes. The non-ballistic model, as outlined before, can explain the observed phenomena. When interpreted in the context of the ballistic model, the two sharp apparent bends of 90 deg should move outward as a whole relative to the core. Since each component in the staircase mode corresponds to a knot ejected previously, it should not have remained stationary. The staircase mode may induce pressure gradients in the medium through which the jet propagates, triggered by its own interaction with the ambient medium. In this case, gradual changes in the position and curvature of the jet bends near K8 and K6 with time can be expected (Gómez et al. 2001). Plausibly, such a scenario could be responsible for the small angle variations of the jet. However, to explain the two 90 degree bends would require very special conditions in the ambient medium, making it hard to understand why such an ambient medium existed only during 1992-2000, but was absent before mid-1992 and again after 2001. As pointed out by Gómez et al. (2001), the moving-component scenario suggests very nonballistic trajectories for K5 and K6. But there are still unanswered questions concerning the origin of these non-ballistic trajectories. Are they caused by continuous or discret ejection events, and what role, if any, is played by the jet precession? Further detailed investigations and modeling is required to answer these questions.

\subsection{Timescales: flux-density variability and mode change}

The three drastic mode changes are clearly correlated with maxima in the optical light-curve. Thus we assume that the optical emission (most likely emergent from the core) causes the change in the trajectory. In two cases, a major long-term radio flux 
outburst occurs shortly after a mode change has appeared and this can be explained within the non-ballistic motion scenario of hotspots outlined in the previous subsection. In the optical emission, Fan et al. (1997) and Qian \& Tao (2004) found periodic features of 13.8-14.2 years. This is roughly twice the time interval we find here between the two mode transitions. A physical connection between the morphological changes (mode changes) and the flux variability in the radio and the optical regime is thus likely.

According to conventional wisdom, radio outbursts are explained as correlated with the ejection of a new radio component from the core. However, in PKS $0735+178$ we found a similar number of components over a time span of roughly 14 years and no new components were unequivocally detected during this period. In addition, we find a correlation with the morphological changes described as mode changes. Furthermore, we are able to explain the flux-density changes within the model by Gong et al. (2008) of non-ballistically moving hotspots. Thus, it can be inferred that in PKS 0735+178 ejection of VLBI components from the core is not required to explain the observed (quasi-periodic) radio flaring.

We found similar results for the other well-known blazars, $1803+784$ and $0716+714$ - the long-term radio variability can be correlated with morphological changes in the nuclear jet and is hence also likely to be of geometric origin. We thus find in three BL Lac objects supporting evidence for a correlation between long-term radio variability and morphological changes that are likely to be of geometric origin. Additional contribution to morphological changes, particularly the more abrupt ones, could come from short bursts of power injected into the jet by the central engine, manifested by the optical flaring events.

\section{Conclusions}

- We report a third (and the most clear) event of structural mode transition in the nuclear jet of the BL Lac object PKS 0735+178. In particular, we find that the twice sharply bent trajectory of the nuclear jet transformed into a straight jet sometime between 200.4 and 2001.8.

- From a literature search we further found that a similar transition from a kinky to a straight jet is present in the VLBI observations of this source performed at $5 \mathrm{GHz}$ in December 1981 and June 1983.

- Contrasting kinematic properties are found associated with the two morphological modes of the nuclear jet: in the staircase mode apparent superluminal motion of the knots in the jets is observed, either directed away from the core or towards it. In the straight mode, however, the knots remain fairly stationary relative to the core. Their position angle distribution is broad in the former case $\left(\sim 90^{\circ}\right)$ and narrow in the latter $\left(\sim 20^{\circ}\right)$.

- The time interval between the mode changes is $\sim 11.3$ years between the first two mode changes and $\sim 7.2$ years between the last two mode changes.

- Both mode changes, from kinky to straight jet, are found to coincide with individual, well-defined peaks in the optical light-curve. This points to a causal connection between the optical flaring and the structural changes in the nuclear jet.

- The last two mode changes are seen to occur shortly before major radio outbursts.
- Remarkably, no new radio knot/component was observed during the entire time span (1995.27 to 2008.91) covered by the VLBI images presented in this study - the components in the staircase-mode are fairly unambiguously identifiable with the set of components witnessesd in the straight mode. A smooth transition was shown in this paper, which could be explained by a (most likely curling) jet with a changing viewing angle.

- The kinematics of the VLBI knots were modeled within the non-ballistic motion scenario (Gong et al. 2008).

- The long-term radio flux variability can be explained by Doppler boosting effects arising from the precession of the nuclear jet.

- The model of non-ballistic motion of hotspots explains the variability of flux-density and morphology, the significant morphological changes and the unusual kinematics of the nuclear jet in PKS0735+178.

- Similar kinematic properties - fairly stationary components - and a correlation between periodic ridge line changes and the long-term radio outbursts were found by some of us for two other well-known blazars: $1803+784$ and $0716+714$.

Acknowledgements. This research made use of data from the MOJAVE database that is maintained by the MOJAVE team. This research made use of data from the University of Michigan Radio Astronomy Observatory, which is supported by the National Science Foundation and by funds from the University of Michigan. This research made use of the NASA/IPAC Extragalactic Database (NED) which is operated by the Jet Propulsion Laboratory, California Institute of Technology, under contract with the National Aeronautics and Space Administration. The National Radio Astronomy Observatory is a facility of the National Science Foundation operated under cooperative agreement by Associated Universities, Inc. The research of the authors Biping Gong and J.W. Zhang is supported in part by the National Natural Science Foundation of China, under grands 10778712.

\section{References}

Abraham, R. G., McHardy, I. M., \& Crawford, C. S. 1991, MNRAS, 252, 482 Agudo, I., Gómez, J. L., Gabuzda, D. C., et al. 2006 A\&A, 453, 477 Bååth, L. B., \& Zhang, F. J. 1991, A\&A, 243, 328

Britzen, S., Vermeulen, R. C., Campbell, R. M., et al. 2008, A\&A, 484, 119 Britzen, S., Kam, V. A., Witzel, A., et al. 2009, A\&A, 508, 1205

Britzen, S., Kudryavtseva, N. A., Witzel, A., et al. 2010, A\&A, 511, A57 Ciprini, S., Takalo, L. O., Tosti, G., et al. 2007, A\&A, 467, 465 Cotton, W. D., Wittels, J. J., Shapiro, I. I., et al. 1980, ApJ, 238, L123 Fan, J. H., Xie, G. Z., Lin, R., et al. 1997, A\&AS, 125, 525 Gabuzda, D. C., Wardle, J. F. C., Roberts, D. H., et al. 1994, ApJ, 435, 128 Gómez, J. L., Guirado, J. C., Agudo, I., et al. 2001, MNRAS, 328, 873 Gong, B. P. 2008, MNRAS, 389, 315

Gopal-Krishna, Stalin, C. S., Sagar, R., et al. 2003, ApJ, 586, L25 Gopal-Krishna, Dhurde, S., \& Wiita, P. J. 2004, ApJ, 615, L81 Goyal, A., Gopal-Krishna, Anupama, C., et al. 2009, MNRAS, 399, 1622 Gupta, A. C., Fan, J. H., Bai, J. M., et al. 2008, AJ, 135, 1384

Lister, M. L., Aller, H. D., Aller, M. F, et al. 2009, AJ, 137, 3718

Nakamura, M., \& Meier, D. L. 2009, proceedings of Approaching MicroArcsecond Resolution with VSOP-2: Astrophysics and Technology, ed. Y. Hagiwara, E. Fomalont, M. Tsuboi, \& Y. Murata, ASP Conf. Ser., 402, 357 Piner, Bhattarai, D., Edwards, P. G., Jones, D. L. 2006, ApJ, 640, 196

Pursimo, T., Nilsson, K., Sillanpää, A., et al. 1999, in Proceedings of the BL Lac Phenomenon Conference, ed. L. O. Takalo, \& A. Sillanpää, ASP Conf. Ser., 159,385

Qian, B., \& Tao, J. 2004, PASP, 116, 161

Sbarufatti, B., Treves, A., \& Falomo, R. 2005, ApJ, 635, 173

Scarpa, R., Urry, C. M., Falomo, R., Pesce, J. E., \& Treves, A. 2000, ApJ, 532, 740

Spergel, D. N., Verde, L., Peiris, H. V., et al. 2003, ApJS, 148, 175

Stickel, M., Fried, J. W., \& Kühr, H. 1993, A\&AS, 98, 393 